\title{
Controlling Micron and Submicron Scale Laser Induced Surface Struc- tures on Stainless Steel with Industrial Femtosecond Lasers
}

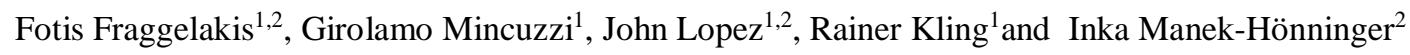 \\ ${ }^{1}$ ALPhANOV, Technological Centre for Optics and Lasers, Optics Institute of Aquitaine, Rue F. Mit- \\ terrand 33400 Talence, France \\ E-mail: fotis.fraggelakis@alphanov.com \\ ${ }^{2}$ Université de Bordeaux, CNRS, CEA, CELIA UMR5107, 33405 Talence, France
}

\begin{abstract}
Generation of laser induced surface structures has been reported as an effective way to functionalize the surface of a solid material. Structure size, symmetry and hierarchical length scale formation has a significant influence on the material's macroscopic properties. The feasibility to produce surface morphologies at different scales is on high demand since they can be employed to mimic bio inspired morphologies in order to enable a variety of unexplored surface functionalities such as bactericidal, anti-icing and anti-reflective surfaces. In the present paper, we report on a comprehensive study dealing with laser induced surface structures morphology control, in terms of symmetry and size, utilizing a high throughput industrial femtosecond source with pulse duration of 350 fs. Interestingly, structures at the micron scale can be effectively tuned via fluence manipulation meanwhile tuning structures at the submicron scale requires double pulse irradiation with polarization modification. For double pulse irradiation the effect of polarization, fluence and overlap was investigated for interpulse delays up to $4 \mathrm{~ns}$. We find out that a large variety of surface structures can be achieved by this way. We believe that our work provides novel data on surface texturing and pushes forward the level of technological readiness of laser surface functionalization.

DOI: $10.2961 / \mathrm{j} \mathrm{lmn} .2018 .03 .0010$
\end{abstract}

Keywords: femtosecond, micro/nanostructuring, 2D-LIPSS, spikes, surface processing, industrial laser processing

\section{Introduction}

Surface texturing with laser induced surface structures has been emerged as a reliable way to induce functionalization on solid surfaces. Several works refer to modifications of optical properties [1], changes in the tribological performance [2], modification of the surface wettability [3] and selective bacterial adhesion [4].

Laser induced periodic surface structures (LIPSS) have been successfully produced on a wide variety of materials ranging from semiconductors [5] to metals [6] and transparent materials [7]. Laser induced surface structure formation results from the combination of several mechanisms. Indeed, for femtosecond laser irradiation in semiconductors [8] and metals [9], electrons absorb a part of the laser pulse energy. The absorbed energy is transferred to the lattice via electron-phonon coupling within a few picoseconds [9-12]. After several tens to hundreds of picoseconds thermal and structural changes take place on the melted surface due to microfluidic movements [10].

Although LIPSS formation has been widely reported in the scientific literature, the mechanism is not fully understood up to now. Morphological characteristics such as the period, the depth and structure homogeneity are predominantly determined by the laser wavelength, polarization, fluence, number of incident pulses $[13,14]$ as well as the spot size [15].

Spike formation results from multi-pulse irradiation with sufficient fluence; the feature size is generally in the range of tens of $\mu \mathrm{m}$. Theoretical investigations show that spike formation results from Marangoni flows due to high ther- mal gradients during laser irradiation [13]. Spikes have been observed in Silicon, amorphous materials [16] and steel processed with infrared, visible [17] or ultraviolet laser irradiation [18]. Furthermore, it has been shown that the spike size can be controlled by tuning heat accumulation effects during laser irradiation [19].

Nevertheless, several functional surfaces, either found in nature [20] or produced by techniques [21] exploit 2D features in the submicron regime. A significant improvement of control over the laser induced structures morphology needs to be acquired in order to fabricate surfaces below a few microns. The generation of two-dimensional submicron periodic features was realized up to date with circular or cylindrical vector beams by a fine tuning of process parameters [22,23]. Here we utilized double pulse irradiation to increase the variety of the produced 2D submicron structures and control their size and symmetry.

Surface structuring under double pulse irradiation exploits the transient surface state to control the resulting morphology. Interestingly, the correlation between the morphological characteristics and the interpulse delay is demonstrated for the interpulse delay regime extending from $\Delta \tau=0.5 \mathrm{ps}$ to $\Delta \tau=4 \mathrm{~ns}$. In the following paragraphs, an overview of our work in controlling the laser induced structures size and morphological characteristics is presented. Features extending from several tens of microns in size down to the nanometre scale are shown.

\section{Experimental setup}

2.1 Trains of single pulse at high repetition rates 
For the spike generation, an industrial femtosecond laser source emitting at $1030 \mathrm{~nm}$ (Amplitude, Tangerine) was utilized to structure the surface of $316 \mathrm{~L}$ polished stainless steel samples with $0.5 \mathrm{~mm}$ thickness commercially available from RS. The samples were not cleaned before irradiation. The pulse duration was $350 \mathrm{fs}$, and the repetition rate was set to $1 \mathrm{MHz}$. A $100 \mathrm{~mm}$ - $\mathrm{f}$ (theta) lens focuses the beam to a spot diameter of $2 \omega_{0}=35 \mu \mathrm{m}$, measured with a beam profiler. The key process parameters such as the repetition rate (f), the fluence $(\Phi)$, the number of scans $(\mathrm{N})$ and the pulses per spot (pps) as defined in [19], were varied in a systematic way. The values used in the experiment are detailed in Table 1 . The surface was investigated using scanning electron microscopy (SEM) analysis. The spike size was extracted from SEM pictures applying the open source software Gwyddion.

Table 1 Process parameters and values considered in the single pulse experiments.

\begin{tabular}{|cc|}
\hline Repetition rate, $\mathrm{f}[\mathrm{kHz}]:$ & 1000 \\
\hline \multirow{2}{*}{ Fluence, $\Phi\left[\mathrm{J} / \mathrm{cm}^{2}\right]:$} & $0.04,0.11,0.22$, \\
& $0.27,0.37,0.42,0.54$ \\
\hline Number of scans, [N]: & 50 \\
\hline Pulses per spot [pps]: & 15 \\
\hline
\end{tabular}

\subsection{Double pulse irradiation}

We have used a 350 fs-pulse duration ultrafast laser emitting at $1030 \mathrm{~nm}$ and operating at $100 \mathrm{kHz}$ for double pulse experiments. A delay line setup was implemented and described in detail elsewhere [24]. It is designed to generate double cross-polarized (XP) and double counter rotating circularly polarized (CP) pulses with variable interpulse delays. This setup enables us to exploit almost $100 \%$ of the available laser power. The interpulse delay $(\Delta \tau)$, which is the time interval between two cross-polarized pulses having the same fluence, was increased up to 4 ns with an uncertainty smaller than 10 ps. The beam was focused by a $100 \mathrm{~mm}$ - $\mathrm{f}$ (theta) lens to a $25 \mu \mathrm{m}$ spot size $\left(2 \omega_{0}\right.$ at $\left.1 / \mathrm{e}^{2}\right)$. The fluence $(\Phi)$, the pulses per spot (pps) and the hatch $(\mathrm{H})$ were set in order to optimize the surface morphology; the values considered are shown in Table 2 . We have textured surfaces up to $1.3 \mathrm{~mm}$ x $0.6 \mathrm{~mm}$. SEM micrographs were subjected to Fast Fourier Transformation using Gwyddion in order to evaluate the homogeneity and periodicity of the generated structures.

Table 2 Process parameters and values considered in the double pulse experiment.

\begin{tabular}{|cc|}
\hline Fluence, $\Phi\left[\mathrm{J} / \mathrm{cm}^{2}\right]:$ & $0.1,0.15,0.2$, \\
\hline Overlap $[\mathrm{pps}]:$ & $5,10,20$, \\
\hline Hach, $\mathrm{H}[\mu \mathrm{m}]:$ & $1,2,5$, \\
\hline & $\pm 0.1, \pm 0.2, \pm 0.5, \pm 1, \pm 2, \pm 5$ \\
Interpulse delay $(\Delta \tau)$ & $\pm 10, \pm 20, \pm 50, \pm 100, \pm 200, \pm$ \\
{$[\mathrm{ps}]:$} & $500, \pm 1000, \pm 2000, \pm 3000$ \\
\hline
\end{tabular}

\section{Results and discussion}

\subsection{Controlling spike size}

It has been shown that for low repetition rate processing ( $\mathrm{f}<1 \mathrm{kHz}$ ), the fluence value has a significant influence on the spike size. Indeed, the spike size rises from $\delta$ $=6 \mu \mathrm{m}$ to $\delta=24 \mu \mathrm{m}$ while increasing the fluence from $\Phi=$ 0.5 to $\Phi=1.2 \mathrm{~J} / \mathrm{cm}^{2}$ [25]. When the repetition rate exceeds
$500 \mathrm{kHz}$, the interpulse time interval is not sufficient for the material to dissipate the heat load away from the surface, thus the surface temperature increases slowly pulse after pulse. This phenomenon, referred to as heat accumulation [26], affects the spikes formation since it is a result of temperature-sensitive, molten material motion. Parameters that affect the accumulated heat, such as fluence, pps and repetition rate, can be correlated to the spike size. At 1 $\mathrm{MHz}$, the spike size increases from $\delta=8 \pm 2 \mu \mathrm{m}$ to $\delta=59$ $\pm 15 \mu \mathrm{m}$ when the fluence increases from $\Phi=0.16 \mathrm{~J} / \mathrm{cm}^{2}$ to $\Phi=0.54 \mathrm{~J} / \mathrm{cm}^{2}$. Corresponding spikes are depicted in Fig.1.
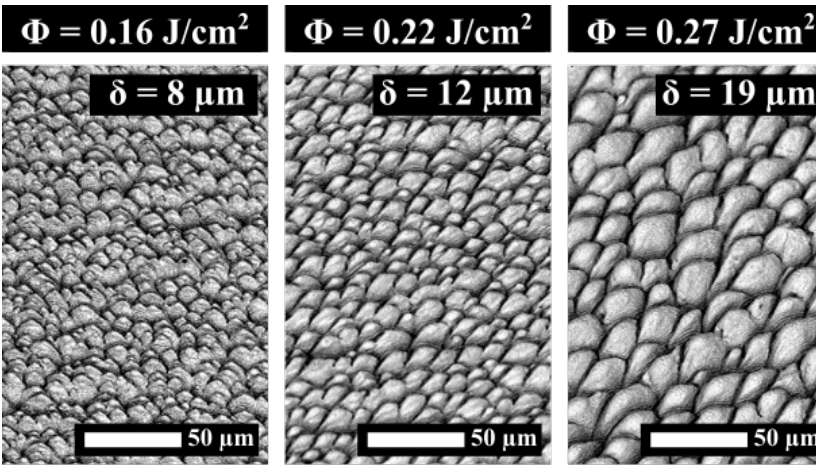

$\Phi=0.34 \mathrm{~J} / \mathrm{cm}^{2}$
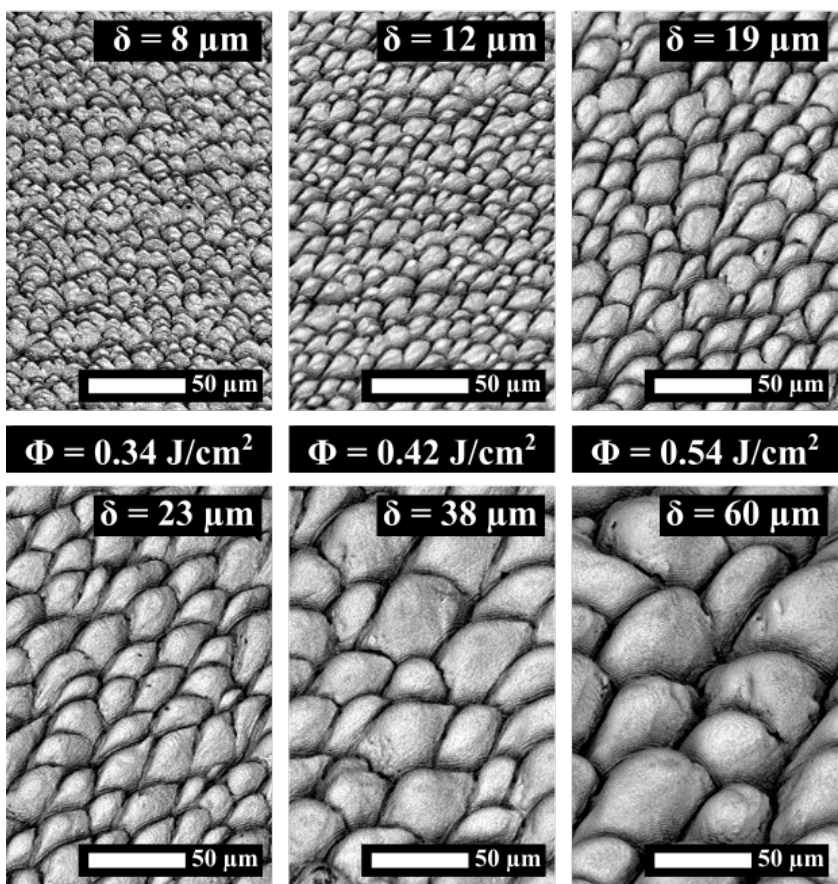

$\Phi=0.54 \mathrm{~J} / \mathrm{cm}^{2}$

Fig.1 SEM images of spikes obtained with different fluences when pps $=70$ and $\mathrm{N}=50$.

Nevertheless, the decrease of the spike size below a few microns cannot be realized by means of fluence variation. In the case of $1 \mathrm{MHz}$, a fluence threshold for spike formation was observed for $\Phi=0.16 \mathrm{~J} / \mathrm{cm}^{2}$ which yields a spike size of $\delta=8 \pm 2 \mu \mathrm{m}$ [19].

\subsection{Two dimensional LIPSS}

In order to overcome the size limitations of 2D surface structures, we investigated the double pulse approach to control submicron morphologies. The generation of structures other than ripples with sizes below $1 \mu \mathrm{m}$ requires a fine tuning of the pulse-to-pulse overlap and the polarization [22,23]. Double pulse irradiation can be employed the control the structures symmetry and size producing a variety of novel 2D submicron structures. In the present work, we have used XP pulses and CP pulses to texture the sample surface. The interpulse delay was varied between 0 ps and 4 ns as described in section 2.2

Surface morphologies obtained for overlap pps $=10$ and hatch $\mathrm{H}=1 \mu \mathrm{m}$ at a fluence of $\Phi=0.1 \mathrm{~J} / \mathrm{cm}^{2}$ are depicted in Fig. 2 for different interpulse delays $(\Delta \tau)$. Homogeneous triangular structures were obtained for $\Delta \tau=0.5$ ps and $\Delta \tau=$ 2 ps, meanwhile chaotic and inhomogeneous structures are 
obtained with $\Delta \tau=10 \mathrm{ps}$ and $\Delta \tau=100 \mathrm{ps}$. The surface structures become again homogeneous for $\Delta \tau=500 \mathrm{ps}$, when ripples are formed perpendicular to the first pulse polarization. Finally, rhombic structures appear on the surface for $\Delta \tau=3 \mathrm{~ns}$.

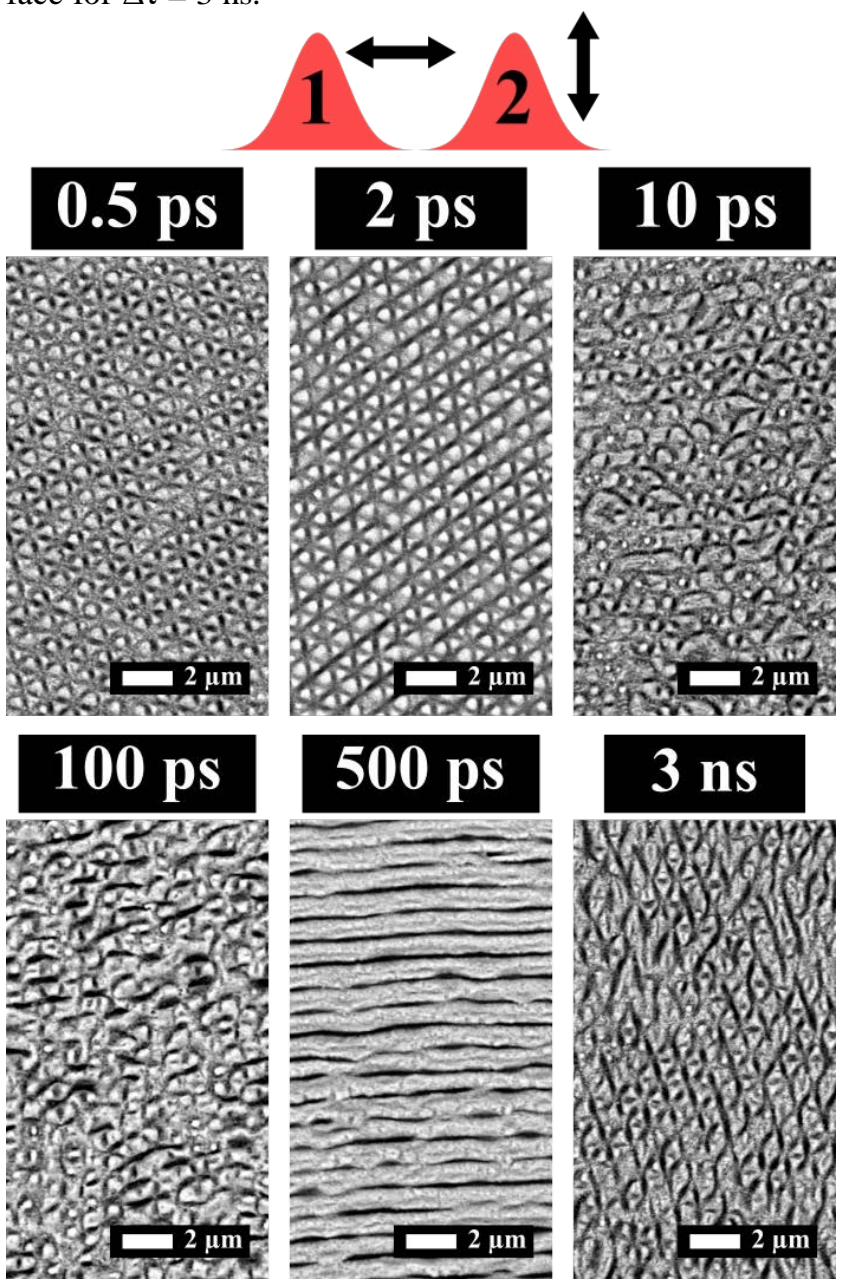

Fig.2: SEM images of stainless steel surface processed with pps $=10, \mathrm{H}=1 \mu \mathrm{m}$ and different interpulse delay values. The fluence value was fixed to $\Phi=0.1 \mathrm{~J} / \mathrm{cm}^{2}$.

The differences in the obtained structures for varying delays can be linked to the different stages of ultrafast evolution of the excited surface state. Indeed, four distinctive time delay zones can be identified. The first one refers to $\Delta \tau=0.5 \mathrm{ps}$ and $\Delta \tau=2 \mathrm{ps}$, where homogeneous triangles were obtained. Within this timescale, the electrons are equilibrating with the lattice and yet, no structural effects are expected on the surface $[27,28]$. The second zone extends from $\Delta \tau=10 \mathrm{ps}$ to $\Delta \tau=100 \mathrm{ps}$, where the structures are disordered. Within this timescale, surface expansion and ablation [29] are expected to occur, and laser induced features are expected to form on the surface [27]. The third zone refers to $\Delta \tau=500 \mathrm{ps}$, for which ripples were obtained on the surface. At this timescale, the surface is considered to be melted and features which are induced by the first pulse should be prominent on the surface [27]. Last but not least, in the fourth zone, when $\Delta \tau=3$ ns the interpulse delay is comparable with the resolidification time, therefore the lattice structure reappears, and the second pulse inter- acts again with the surface under the same conditions as the first one. As a result, the structures obtained for very small delays ( $\Delta \tau=0.5 \mathrm{ps}$ and $\Delta \tau=2 \mathrm{ps}$ ) and very large delays ( $\Delta \tau=3 \mathrm{~ns})$ exhibit similarities.

The period of the structures obtained with pps $=10, \mathrm{H}=1$ $\mu \mathrm{m}, \Phi=0.1 \mathrm{~J} / \mathrm{cm}^{2}$ at a delay of $\Delta \tau=3 \mathrm{ps}$ was extracted with FFT analysis of the SEM image. The SEM as well as the FFT image are shown in Fig. 3 left and Fig. 3 right, respectively. The periods of the structures along the three different axes of symmetry, which are indicated with orange arrows, are $955 \pm 4 \mathrm{~nm}, 870 \pm 3 \mathrm{~nm}$ and $856 \pm 3 \mathrm{~nm}$, respectively. The periods are consistent with those of $1 \mathrm{D}$ LSFL obtained on stainless steel at similar conditions [12]. The angles between the axes of symmetry are 62, 65 and 53 degrees.

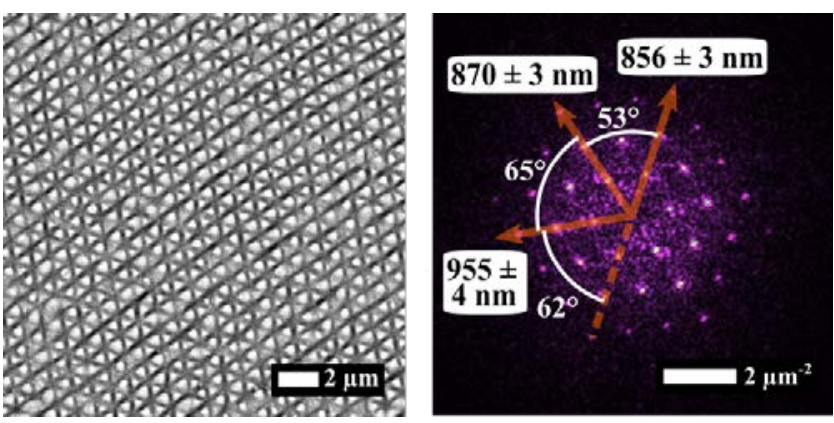

Fig.3 Left: SEM image of surface obtained with pps $=10, \mathrm{H}=1$ $\mu \mathrm{m}, \Phi=0.1 \mathrm{~J} / \mathrm{cm}^{2}$ at a delay of $\Delta \tau=3$ ps. Right: Fast Fourier Transformation of SEM image showing the three axes of symmetry, the angles between them and the period of the structures for each axis are indicated.

\subsection{Biomimetic surface obtained with CP pulses in the submicron regime}

Breaking the barrier for generating laser induced structures in the nano scale is an ever-present challenge. When the material is processed with linear polarized femtosecond pulses with fluence values below the ripple formation threshold, high spatial frequency LIPSS (HSFL) can be obtained oriented parallel to the laser polarization [30]. Moreover, for double pulse irradiation near the LIPSS formation threshold a systematic increase of the HSFL area has been reported as a function of increasing $\Delta \tau$ up to 5 ps [31]. In our work, extending the interpulse delay window to $\Delta \tau=20$ ps we obtain HSFL after irradiating the stainless steel surface with double counter rotating circularly polarized pulses. For $\Phi=0.1 \mathrm{~J} / \mathrm{cm}^{2}, \mathrm{H}=1 \mu \mathrm{m}$ and for overlap values which were varied between pps $=5$ and pps $=50$. In contrary to the single pulse case, the HSFL obtained with double pulse irradiation evolves with increasing overlap. In Fig.4 (left) we demonstrate this effect, SEM images of the obtained HSFL are illustrated with respect to the pps value. High frequency ripples constitute a 2D surface morphology (2D-HSFL).

Fourier analysis on the SEM image of Fig. 4 with $\Phi=$ $0.1 \mathrm{~J} / \mathrm{cm}^{2}, \Delta \tau=20 \mathrm{ps}, \mathrm{H}=1 \mu \mathrm{m}$ and $\mathrm{pps}=5$ gives an average spatial period between the HSFL $\mathrm{T}_{\text {average }}=284 \pm 8 \mathrm{~nm}$. 


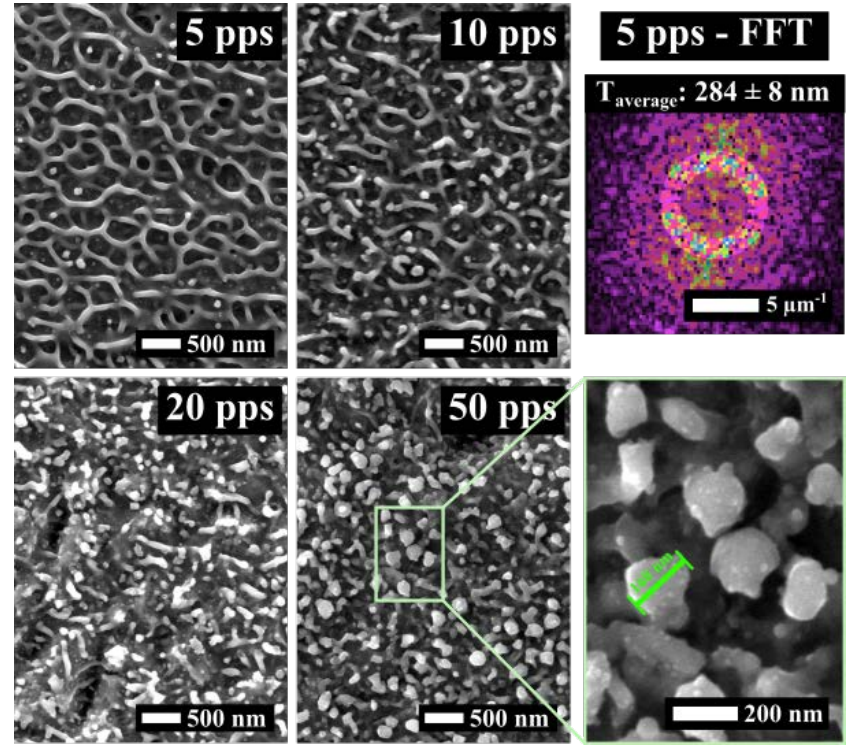

Fig.4 Left: SEM images of the nanostructure obtained with CP pulses and variable pps as indicated. $\Phi=0.1 \mathrm{~J} / \mathrm{cm}^{2}, \Delta \tau=20 \mathrm{ps}$ and $\mathrm{H}=1 \mu \mathrm{m}$. Right top: Fourier transformation of SEM image for pps $=5$. Right bottom: close up of SEM image of the structures obtained for pps $=50$.

In contrast to Fig. 3 no preferred axis of symmetry can be identified. Strikingly, for pps $=50$ the obtained morphology is similar to the morphology of the structuring found on the dragonfly (Rhyothemis phyllis chloe) wing membrane as shown in [20]. Grain analysis distribution over 321 grains for the case of $\Phi=0.1 \mathrm{~J} / \mathrm{cm}^{2}, \Delta \tau=20 \mathrm{ps}, \mathrm{H}=1 \mu \mathrm{m}$ and pps $=50$, gives an average grain diameter $\delta_{\text {average }}=146$ $\pm 50 \mathrm{~nm}$.

\section{Conclusions}

We presented several efficient strategies to control twodimensional micro and nano morphologies on stainless steel surface in different scales with direct femtosecond laser texturing. We showed that the spike size in the microscale can be tuned with the fluence. Typical spikes ranging from $\delta=8 \pm 2 \mu \mathrm{m}$ to $\delta=59 \pm 15 \mu \mathrm{m}$ were obtained by this means. Furthermore, double pulse irradiation induced 2D structures on a submicron scale. For instance, homogeneous submicron triangular structures 2D-LIPSS were realized owing to double cross-polarized pulses, whereas random 2D-HSFL with sizes ranging in the nanoscale were obtained using double counter rotating circularly polarized pulses. We are confident that our data provide novel insight into controlling laser induced surface nanostructures and pave the way for their industrial implementation.

\section{Acknowledgments}

The authors would like to thank Horizon 2020 Framework Programme (H2020) under the Marie Skłodowska-Curie grant agreement (No 675063) for funding this research.

\section{References}

[1] A. Y. Vorobyev and C. Guo: Appl. Phys. Lett., 92, (2008) 1.

[2] J. Bonse, S. V Kirner, M. Gripentrog, D. Spaltmann, and J. Krüger: Materials (Basel)., 11, (2018) 801.
[3] V. Zorba, E. Stratakis, M. Barberoglou, E. Spanakis, P. Tzanetakis, S. H. Anastasiadis, and C. Fotakis: Adv. Mater., 20, (2008) 4049.

[4] C. Simitzi, P. Efstathopoulos, A. Kourgiantaki, A. Ranella, I. Charalampopoulos, C. Fotakis, I. Athanassakis, E. Stratakis, and A. Gravanis: Biomaterials, 67, (2015) 115.

[5] J. Bonse, S. Baudach, J. Krüger, W. Kautek, and M. Lenzner: Appl. Phys. A Mater. Sci. Process., 74, (2002) 19.

[6] G. Tsibidis, E. Skoulas, and E. Stratakis: Opt. Lett., 40, (2015) 5172.

[7] A. Papadopoulos, E. Skoulas, G. D. Tsibidis, and E. Stratakis: Appl. Phys. A, 124, (2018) 146.

[8] S. K. Sundaram and E. Mazur: Nat. Mater., 1, (2002) 217.

[9] M. Bonn, D. N. Denzler, S. Funk, M. Wolf, S.-S. Wellershoff, and J. Hohlfeld: Phys. Rev. B, 61, (2000) 1101.

[10] G. D. Tsibidis, M. Barberoglou, P. A. Loukakos, E. Stratakis, and C. Fotakis: Phys. Rev. B, 86, (2012) 115316.

[11] B. Rethfeld, A. Kaiser, M. Vicanek, and G. Simon: Phys. Rev. B, 65, (2002) 214303.

[12] G. D. Tsibidis, A. Mimidis, E. Skoulas, S. V Kirner, J. Krüger, J. Bonse, and E. Stratakis: Appl. Phys. A, 124, (2018) 27.

[13] G. D. Tsibidis, C. Fotakis, and E. Stratakis: Phys. Rev. B, 92, (2015) 041405.

[14] O. Varlamova, M. Bounhalli, and J. Reif: Appl. Surf. Sci., 278, (2013) 62.

[15] I. Gnilitskyi, T. J. Derrien, Y. Levy, N. M. Bulgakova, and L. Orazi: Sci. Rep., 7, (2017).

[16] E. Peng, A. Tsubaki, C. A. Zuhlke, M. Wang, R. Bell, M. J. Lucis, T. P. Anderson, D. R. Alexander, G. Gogos, and J. E. Shield: Appl. Surf. Sci., 396, (2016) 1170 .

[17] F. Fraggelakis, G. Mincuzzi, J. Lopez, I. Manekhönninger, and R. Kling: Proc. SPIE, 10092, (2017) 1.

[18] F. Fraggelakis, G. Mincuzzi, I. Manek-Hönninger, J. Jopez, and R. Kling: RSC Adv., 8, (2018) 16082.

[19] F. Fraggelakis, G. Mincuzzi, J. Lopez, I. ManekHönninger, and R. Kling: Opt. Express, 25, (2017) 18131.

[20] H. Hu, J. A. Watson, B. W. Cribb, and G. S. Watson: Biofouling, 27, (2012) 37.

[21] A. Elbourne, R. J. Crawford, and E. P. Ivanova: J. Colloid Interface Sci., 508, (2017) 603.

[22] E. Skoulas, A. Manousaki, C. Fotakis, and E. Stratakis: Sci. Rep., (2017) 45114.

[23] J. M. Romano, A. Garcia-Giron, P. Penchev, S. S. S. Dimov, A. G. Giron, P. Penchev, and S. S. S. Dimov: Appl. Surf. Sci., 440, (2018) 162.

[24] F. Fraggelakis, G. Mincuzzi, J. Lopez, I. Manek-, and R. Kling: Proc. SPIE, (2018).

[25] B. K. Nayak and M. C. Gupta: Opt. Lasers Eng., 48, (2010) 940.

[26] F. Bauer, A. Michalowski, T. Kiedrowski, and S. Nolte: Opt. Express, 23, (2015) 1035.

[27] X. Jia, T. Q. Jia, N. N. Peng, D. H. Feng, S. A. Zhang, and Z. R. Sun: J. Appl. Phys., 115, (2014).

[28] J. Reif, O. Varlamova, M. Bounhalli, M. Muth, and T. 
Arguirov: Appl. Surf. Sci., 258, (2012) 9491.

[29] K. S. Tinten, A. Barty, S. Boutet, U. Shymanovich, H. Chapmanb, M. Bogan, S. Marchesini, S. Hau-Riege, N. Stojanovic, J. Bonse, Y. Rosandi, H. M. Urbassek, R. Tobey, H. Ehrke, A. Cavalleri, S. Düsterer, H. Redlin, M. Frank, S. Bajt, J. Schulz, M. Seibert, J.
Hajdu, R. Treusch, C. Bostedt, M. Hoener, and T. Möller: AIP Conf. Proc., (2010).

[30] J. Bonse, S. Höhm, A. Rosenfeld, and J. Kruger: Appl. Phys. A Mater. Sci. Process., 110, (2013) 547.

[31] S. Höhm, A. Rosenfeld, J. Kruger, and J. Bonse: Appl. Surf. Sci., 278, (2013) 7.

(Received: June 16, 2018, Accepted: October 13, 2018) 